\title{
Local Normality Properties of Some Infrared Representations ${ }^{\star}$
}

\author{
Sergio Doplicher and Mauro Spera $\star \star$ \\ Istituto. Matematico "G. Castelnuovo", Università di Roma, I-00185 Roma, Italy
}

\begin{abstract}
We consider the positive energy representations of the algebra of quasilocal observables for the free massless Majorana field described in preceding papers $[1,2]$. We show that by an appropriate choice of the (partially) occupied one particle modes we can find irreducible, type $\mathrm{II}_{\infty}$ or $\mathrm{III}_{\lambda}$ representations in this class which are unitarily equivalent to the vacuum representation when restricted to any forward light cone and disjoint from it when restricted to any backward light cone, or conversely. We give an elementary explicit proof of local normality of each representation in the above class.
\end{abstract}

\section{Introduction}

If $\Phi_{1}, \Phi_{2}, \ldots$ is a sequence of one particle states of a free massless Majorana particle with compact and mutually disjoint supports in momentum space and such that the series of their energy upper bounds is convergent, we can define a non-Fock irreducible representation of the free massless Majorana field, induced by a state where each mode $\Phi_{1}, \Phi_{2}, \ldots$ is occupied and each orthogonal mode is empty. These representations are then covariant under space time translations and obey the spectrum condition [1].

We showed in [2] that the state where each mode $\Phi_{1}, \Phi_{2}, \ldots$ is occupied with probability $\lambda, 0<\lambda<1$, and each orthogonal mode is empty, induces a representation of the algebra of quasilocal observables of the free Majorana massless field which is also covariant and obeys the spectrum condition, and generates a von Neumann algebra which is a type $\mathrm{II}_{\infty}$ factor if $\lambda=\frac{1}{2}$ and a type $\mathrm{III}_{\mu}$ factor, $\mu$ or $\mu^{-1}$ $=(\lambda /(1-\lambda))^{2}$ otherwise. (Actually all properly infinite, injective von Neumann algebras with separable predual can appear in positive energy representations of this model [2].)

Here we discuss these representations in connection with the notion of charge

* Research supported by Ministero della Pubblica Istruzione and C.N.R.-G.N.A.F.A.

$\star \star$ Supported by Istituto Nazionale di Alta Matematica "F. Severi" 
class introduced recently by $D$. Buchholz [3]. Two representations $\pi_{1}, \pi_{2}$ of the $C^{*}$ algebra $\mathfrak{U}$ of quasilocal observables in a theory involving massless particles are said to be in the same charge class if (they are covariant, positive energy and)

$$
\pi_{1}\left|\mathfrak{U}\left(V^{+}\right) \cong \pi_{2}\right| \mathfrak{U}\left(V^{+}\right),
$$

where $\mathfrak{A}\left(V^{+}\right)$is the subalgebra generated by all observables localized in the future light cone $V^{+}$. Although this notion was proposed in [3] for the study of quantum electrodynamics, it might be instructive to test it in our model where univalence is the only superselection rule.

We show that, by an appropriate choice of the one particle modes $\Phi_{1}, \Phi_{2}, \ldots$, we can have the irreducible respectively type $\mathrm{II}_{\infty}$ or $\mathrm{III}_{\mu}$ representations $\pi$ described above fulfilling the following conditions

$$
\begin{aligned}
& \pi\left|\mathfrak{B}\left(V^{+}\right) \cong \pi_{0}\right| \mathfrak{B}\left(V^{+}\right), \\
& \pi\left|\mathfrak{B}\left(V^{-}\right) \downarrow \pi_{0}\right| \mathfrak{B}\left(V^{-}\right),
\end{aligned}
$$

where $\mathfrak{B}(\mathcal{O})$ is the net of local observables for the Majorana field and $\pi_{0}$ is its vacuum representation; the symbol $\delta$ denotes disjointness of representations. Alternatively we can arrange it so that the roles of the future and past light cone are exchanged in (1.2), (1.3); or we can arrange it so that $\pi$ and $\pi_{0}$ are disjoint from the vacuum when restricted to each future or past light cone. One might conjecture that it is impossible, within the mentioned class of models, to find a representation which is normal relative to the vacuum both on $V^{+}$and $V^{-}$. This suggests that the notion of charge class should be modified to the tighter condition

$$
\begin{aligned}
& \pi_{1}\left|\mathfrak{U}\left(V^{+}\right) \cong \pi_{2}\right| \mathfrak{U}\left(V^{+}\right), \\
& \pi_{1}\left|\mathfrak{U}\left(V^{-}\right) \cong \pi_{2}\right| \mathfrak{U}\left(V^{-}\right) ;
\end{aligned}
$$

this possibility was actually left open in [3] and would not essentially modify the discussion given there.

The physical idea behind our construction is very simple. Given any choice of the modes $\Phi_{1}, \Phi_{2},$. with the properties mentioned above, these properties remain unchanged if we translate each $\Phi_{n}$ by a different time translation. If we choose these translations in such a way that the wave packet in space time associated to $\Phi_{n}$ is more and more precisely localized in $V^{-}$(respectively $V^{+}$) as $n \rightarrow \infty$, we will obtain a representation fulfilling the relations (1.2), (1.3) (respectively the converse relations). This is essentially due to the Huygens principle.

We also give in the Appendix a direct argument showing local normality of the explicit models of type II and III representations with positive energy discussed in [2], a property which would follow from more general arguments [4].

The problem of the type of positive energy representations, in the same charge class of the vacuum in the sense of $\left(1.1^{\prime}\right)$, remains open and will be studied elsewhere.

\section{Normality of Light Cones}

Let $\mathscr{H}$ be the Hilbert space of state vectors of a Majorana particle, i.e. the direct sum of the spaces of the irreducible representations of the covering $\widetilde{P}$ of the Poincare 
group of zero mass, spin $\frac{1}{2}$, and helicity \pm . The CAR algebra $\mathfrak{A}(\mathscr{H})$ over $\mathscr{H}$ is generated by the free Majorana field $\Psi$, where

$$
\Psi(f)=a(T f)+a(S f)^{*},
$$

where the destruction operators $a(\Phi), \Phi \in \mathscr{H}$ are generators of $\mathfrak{U}$ fulfilling the CAR, $f$ varies in the test function space $\mathscr{S}\left(\mathbb{R}^{4}\right) \otimes \mathbb{C}^{4}$ of $\mathbb{C}^{4}$-valued $C^{\infty}$ functions with rapid decrease over Minkowski space, and $T, S$ are the appropriate linear respectively antilinear maps of $\mathscr{S}\left(\mathbb{R}^{4}\right) \otimes \mathbb{C}^{4}$ in $\mathscr{H}$ which intertwine the action of $\widetilde{\mathscr{P}}$ on test functions with the unitary representation of $\widetilde{P}$ on $\mathscr{H}$ (see Appendix).

As in $[1,2]$ we define the $C^{*}$ algebra of observables $\mathfrak{B}(\mathcal{O})$ associated to a region $\mathcal{O}$ in space time as the $C^{*}$ algebra generated by the even polynomials in $\{\Psi(f)$; $\operatorname{Supp} f$ $\subset \mathcal{O}\}$. The algebra of quasilocal observables $\mathfrak{B}$ is then the fixed point subalgebra $\mathfrak{U}(\mathscr{H})_{e}$ of $\mathfrak{U}(\mathscr{H})$ under the automorphism induced by $\Phi \in \mathscr{H} \rightarrow-\Phi$.

With $\Phi_{1}, \Phi_{2}, \ldots$ an infinite orthonormal set in $\mathscr{H}$ fulfilling the conditions given at the beginning of Sect. 1, generating the closed subspace $E \mathscr{H}$ of $\mathscr{H}, E$ a self-adjoint projection, the states described in the Introduction are the restriction to $\mathfrak{B}$ of the gauge invariant quasifree state $\omega_{\lambda E}$ of $\mathfrak{U}(\mathscr{H})$ determined by the one particle operator $\lambda E$ (see [2] and ref. therein). These states can be considered as restrictions of pure gauge invariant quasifree states over the CAR algebra $\mathfrak{U}(\mathscr{H} \oplus \mathscr{H})$ [5]. Namely, let

$$
\tilde{\Phi}_{n}=\lambda^{1 / 2} \Phi_{n} \oplus(1-\lambda)^{1 / 2} \Phi_{n^{\prime}} \quad n=1,2, . .,
$$

and let $P_{\lambda}$ be the self-adjoint projection onto the closed subspace of $\mathscr{H} \oplus \mathscr{H}$ generated by $\widetilde{\Phi}_{1}, \tilde{\Phi}_{2}, \ldots \mathfrak{U}(\mathscr{H} \oplus \mathscr{H})$ is generated by the 2-multiplet of Majorana fields

$$
\widetilde{\Psi}: f \oplus g \rightarrow \widetilde{\Psi}(f \oplus g)=a(T f \oplus T g)+a(S f \oplus S g)
$$

(or by the Dirac field $\tilde{\Psi}\left(f \otimes 1 / \sqrt{2}\left(e_{1}+i e_{2}\right)\right)$, with $e_{1}, e_{2}$ any fixed real orthonormal basis in $\left.\mathbb{C}^{2}\right)$. Upon identification of $\Psi(f)$ with $\widetilde{\Psi}(f \oplus 0)$, the restriction to $\mathfrak{B}$ of the pure quasifree state $\omega_{P_{\lambda}}$ of $\mathfrak{U}(\mathscr{H} \oplus \mathscr{H})$ is $\omega_{\lambda E}$ [2]. Note that under the direct sum representation of $\widetilde{P}$ on $\mathscr{H} \oplus \mathscr{H}, \widetilde{\Phi}_{1}, \widetilde{\Phi}_{2}, \ldots$ obey the same conditions as the original set $\Phi_{1}, \Phi_{2}, \ldots$ and the irreducible representations induced by $\omega_{P \lambda}$ on $\mathfrak{U}(\mathscr{H} \oplus \mathscr{H})$ or by its restriction $\tilde{\omega}_{\lambda}$ on $\widetilde{\mathfrak{B}}=\mathfrak{U}(\mathscr{H} \oplus \mathscr{H})_{e}$ are covariant with positive energy. Due to the next lemma it is enough to study the local normality properties of these irreducible representations.

2.1. Lemma. Let $\mathscr{S} \subset \mathbb{R}^{4}$ be any region whose spacelike or timelike complement has nonvoid interior. For each $0 \leqq \lambda \leqq 1$, we have

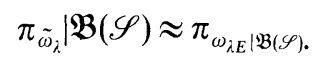

Proof. Since each state $\omega_{\lambda E}, \tilde{\omega}_{\lambda}, \omega_{P_{\lambda}}$ is a restriction of the next one, we have that the GNS representation of $\omega_{\lambda E} \mid \mathfrak{B}(\mathscr{S})$ is a subrepresentation of $\pi_{\omega_{\lambda}} \mid \mathfrak{B}(\mathscr{S})$, which is a subrepresentation of $\pi_{\omega_{P_{\lambda}}} \mid \mathfrak{B}(\mathscr{S})$. To show the quasiequivalence (2.4) it suffices then to show

$$
\pi_{\omega_{P_{\lambda}}} \mid \mathfrak{B}(\mathscr{S}) \approx \pi_{\omega_{\lambda E} \mid \mathfrak{B}(\mathscr{S})}
$$

or that the cyclic projection $E$ in $\pi_{\omega_{\lambda}}(\mathfrak{B}(\mathscr{S}))^{\prime}$ determined by the GNS vector $\tilde{\xi}_{\lambda}$ of $\omega_{P_{\lambda}}$ 
has central support I. This is the case if $\xi_{\lambda}$ is cyclic for $\pi_{\omega_{p},}(\mathfrak{B}(\mathscr{S}))^{\prime}$. If $\mathcal{O}$ is a double cone in the spacelike or timelike complement of $\mathscr{S}$, we have that $\widetilde{\mathfrak{U}}(\mathcal{O})$ and $\mathfrak{B}(\mathscr{S})$ commute, where $\tilde{\mathfrak{A}}(\mathcal{O})$ is the local algebra generated by the Majorana multiplet (2.4), since the anticommutator of $\tilde{\Psi}$ with itself is determined by the kernel $\left(-i \mathbf{S} \gamma_{0} \oplus\right.$ $\left.-i \mathbf{S} \gamma_{0}\right)^{t}$, whose support is the light cone surface $x^{2}=0$ (see Appendix). Since $\xi_{\lambda}$ has compact energy spectrum [1] and $\pi_{\omega_{p}}$ is covariant with positive energy, by the Reeh-Schlieder theorem [6] $\xi_{\lambda}$ is cyclic for $\pi_{\omega_{P_{\lambda}}}(\tilde{\mathfrak{U}}(\mathcal{O}))$ hence also for $\pi_{\omega_{P_{\lambda}}}(\mathfrak{B}(\mathscr{S}))^{\prime}$.

Since the arguments given for the Majorana field (2.1) extend obviously to the multiplet (2.3), it will suffice to prove local normality properties for the pure states $\omega_{E}$ over $\mathfrak{B}$.

2.2. Theorem. We can choose the self-adjoint projector $E$ acting on the one particle Hilbert space $\mathscr{H}$ so that the representations induced by the quasifree state $\omega_{\lambda E}$ on the algebra of quasilocal observable for the Majorana field are all covariant, with positive energy, and quasiequivalent to the vacuum representation when restricted to each future light cone, disjoint from it when restricted to each past light cone. By [2] varying $\lambda$ we obtain all types $I_{\infty}, I I_{\infty}, I I I_{\mu}, 0<\mu<1$.

Proof. By Lemma 2.1, to prove normality on the future light cones it will suffice to discuss the case $\lambda=1$. Let $\psi_{1}, \psi_{2}, \ldots$ be any orthonormal sequence in $\mathscr{H}$ fulfilling the properties of the beginning of Sect. 1. Since the range of the map $f \rightarrow \Psi(f)$ given by $(2.1)$ is dense in $\mathfrak{A}(\mathscr{H})$ and by the anticommutation relations

$$
\|\Psi(f)\|^{2}=\|T f\|^{2}+\|S f\|^{2}=\left(f,\left(-i \mathbf{S} \gamma_{0}\right)^{t} f\right),
$$

using the continuity of the expression (2.6) in the topology of $\mathscr{S}\left(\mathbb{R}^{4}\right)$, we can choose for each $n$ a function $g_{n} \in \mathscr{D}\left(\mathbb{R}^{4}\right) \otimes \mathbb{C}^{4}$ such that

$$
\left\|\Psi\left(g_{n}\right)-a\left(\psi_{n}\right)\right\|<\frac{1}{2^{n}} .
$$

Since $g_{n}$ has compact support, there is a time translation $\tau_{n}$ such that $f_{n} \equiv g_{n \tau_{n}}$ has support in $V^{-}$. With $U$ the unitary representation of the time translations on $\mathscr{H}$, the vectors

$$
U\left(\tau_{n}\right) \psi_{n}=\Phi_{n}
$$

provide an orthonormal sequence in $\mathscr{H}$ with the same properties as above, and the orthogonal projection onto the closed subspace generated by $\Phi_{1}, \Phi_{2}, \ldots$ will be the required projection $E$.

By Lemma 2.1 it is enough to show that $\omega_{E} \mid \mathfrak{B}\left(V^{+}\right)$is a normal state in the vacuum representation of $\mathfrak{B}\left(V^{+}\right)$to prove the quasiequivalence of $\pi_{\omega_{\lambda E}}$ and $\pi_{0}$ when restricted to $\mathfrak{B}\left(V^{+}+a\right)$ for $a=0$ and, by translation covariance, in any $a \in \mathbb{R}^{4}$. Now $\omega_{E}$ is the weak limit of the vector states of the vacuum representation induced by $\xi_{n}$, where

$$
\xi_{n}=a\left(\Phi_{n}\right)_{, 2}^{*} a\left(\Phi_{1}\right)^{*} \Omega .
$$

It suffices to show that the mentioned sequence $\omega_{\xi_{n}} \cdot \pi_{0}$ is norm convergent when 
restricted to $\mathfrak{B}\left(V^{+}\right)$. Since $a(\Phi) \Omega=0$ for each $\Phi \in \mathscr{H}$, by (2.8) we have

$$
\left|\left(\omega_{\xi_{n}}-\omega_{\xi_{n-1}}\right)(B)\right|=\left|\omega_{\xi_{n-1}}\left(a\left(\Phi_{n}\right)\left[B, a\left(\Phi_{n}\right)^{*}\right]\right)\right| \leqq\left\|\left[B, a\left(\Phi_{n}\right)^{*}\right]\right\|
$$

for each $B \in \mathfrak{B}\left(\mathscr{H}_{\pi_{0}}\right)$. By covariance and by (2.7) we have

$$
\left\|\Psi\left(f_{n}\right)-a\left(\Phi_{n}\right)\right\| \leqq \frac{1}{2^{n}},
$$

hence

$$
\left|\left(\omega_{\xi_{n}}-\omega_{\xi_{n-1}}\right)(B)\right| \leqq \frac{1}{2^{n-1}}\|B\|+\left\|\left[B, \Psi\left(f_{n}\right)^{*}\right]\right\| .
$$

If we choose $B \in \pi_{0}\left(\mathfrak{B}\left(V^{+}\right)\right)$, we have $\left\|\left[B, \Psi\left(f_{n}\right)^{*}\right]\right\|=0$ since the field anticommutator has support on the surface of the light cone and $f_{n}$ has support in $V^{-}, B$ is even, then by (2.10) for each $A \in \mathfrak{B}\left(V^{+}\right)$,

$$
\left|\omega_{E}(A)-\omega_{\xi_{n}}\left(\pi_{0}(A)\right)\right| \leqq \frac{1}{2^{n-1}}\|A\|,
$$

and $\omega_{E}$ is normal on $\mathfrak{B}\left(V^{+}\right)$relative to the vacuum representation.

Next we show that the restrictions of $\pi_{\omega_{\lambda E}}$ and $\pi_{0}$ to $\mathfrak{B}\left(V^{-}+a\right)$ are disjoint for $a=0$ hence for each $a$. Since by [3, Lemma 2.1] both restrictions are factorial, it suffices to find a central sequence $\chi_{n} \in \mathfrak{B}\left(V^{-}\right)_{1}$ such that

$$
\lim _{n \rightarrow \infty} \omega_{0}\left(\chi_{n}\right)=0, \quad \lim _{n \rightarrow \infty} \omega_{\lambda E}\left(\chi_{n}\right) \neq 0 .
$$

By definition $\omega_{0}\left(a\left(\Phi_{n}\right) * a\left(\Phi_{n}\right)\right)=0$ and $\omega_{\lambda E}\left(a\left(\Phi_{n}\right)^{*} a\left(\Phi_{n}\right)\right)=\lambda\left(\Phi_{n}, E \Phi_{n}\right)=\lambda$. Since $\Phi_{n}$, $n=1,2, \ldots$, is an infinite orthonormal set, $\left(\Phi_{n}, \Psi\right) \rightarrow 0$ as $n \rightarrow \infty$ for each $\Psi \in \mathscr{H}$ and by the CAR the commutator of $a\left(\Phi_{n}\right)^{*} a\left(\Phi_{n}\right)$ with $B$ tends to zero in norm for $B=a(\Psi)$, $\Psi \in \mathscr{H}$, hence for each $B \in \mathfrak{U}(\mathscr{H})$. Hence by $\left(2.7^{\prime}\right) \lim _{n \rightarrow \infty} \omega_{0}\left(\Psi\left(f_{n}\right) * \Psi\left(f_{n}\right)\right)=0$, $\lim \omega_{\lambda E}\left(\Psi\left(f_{n}\right)^{*} \Psi\left(f_{n}\right)\right)=\lambda,\left\|\Psi\left(f_{n}\right)\right\| \rightarrow 1$. Hence for each $\lambda \neq 0$, the sequence $\stackrel{n \rightarrow \infty}{X}_{n}=\left\|\Psi\left(f_{n}\right)\right\|^{-2} \Psi\left(f_{n}\right)^{*} \Psi\left(f_{n}\right)$ fulfills Eq. (2.11) and belongs to $\mathfrak{B}\left(V^{-}\right)_{1}$.

Remark. In Theorem 2.2, quasiequivalence implies unitary equivalence, e.g. by [3, Proposition 2.3].

\section{Appendix}

By identifying the one particle space $\mathscr{H}$ with the subspace of $L^{2}\left(\mathbb{R}^{3}, d^{3} k\right) \otimes \mathbb{C}^{4}$ of the solutions of the Dirac equation $k \psi(\vec{k})=0, k=(|\vec{k}|, \vec{k})$, or equivalently of the equation $(2|\vec{k}|)^{-1} k \gamma_{0} \psi(\vec{k})=\psi(\vec{k})$, we can define the maps $T, S$ of Eq. (2.1) by

$$
\begin{aligned}
& (T f)(\vec{k})=\sqrt{2 \pi} \frac{k \gamma_{0}}{2|\vec{k}|} C_{1} \hat{f}(|\vec{k}|, \vec{k}), \\
& (S f)(\vec{k})=\sqrt{2 \pi} \frac{k \gamma_{0}}{2|\vec{k}|} \hat{f}(|\vec{k}|, \vec{k}),
\end{aligned}
$$

where $C_{1}$ is the real Pauli matrix such that $C_{1}^{2}=I$ and $C_{1} \gamma_{\mu} C_{1}^{-1}=-g_{\mu \mu} \gamma_{\mu}^{t}$. By Eq. 
(2.6) the map $\Psi$ extends to distributions $F$, where $F(x)=\delta\left(x_{0}\right) f(\vec{x}), f \in L^{2}\left(\mathbb{R}^{3}\right) \otimes \mathbb{C}^{4}$. By choosing $f$ such that $\hat{f}=C_{1} \psi, \psi \in \mathscr{H}$, we have by (A.1) $T F=\psi, S F=0$ by the Dirac equation, and

$$
\Psi(F)=a(\psi)
$$

We conclude by sketching a direct proof of local normality of $\pi_{\omega_{\lambda E}}$ for the generic choice of the orthonormal sequence $\Phi_{1}, \Phi_{2}, \ldots$ generating $E \mathscr{H}$. As in the proof of Theorem 2.1, it suffices to deal with $\lambda=1$ and to prove that the sequence of vector states $\omega_{\xi_{n}} \cdot \pi_{0} \mid \mathfrak{B}(\mathcal{O}), \xi_{n}=a\left(\Phi_{n}\right)^{*} \ldots a\left(\Phi_{1}\right)^{*} \Omega$, is norm convergent for each double cone $\mathcal{O}$ centered at the origin. With $f_{n} \in L^{2}\left(\mathbb{R}^{3}, d^{3} x\right) \otimes \mathbb{C}^{4}$

$$
\hat{f}_{n}=C_{1} \Phi_{n^{\prime}}
$$

as in Eq. (A.2) we identify $a\left(\Phi_{n}\right)$ with the time zero field operator $\Psi\left(F_{n}\right), F_{n}(x)$ $=\delta\left(x_{0}\right) f_{n}(\vec{x})$. We have an estimate as in Sect. 2,

$$
\begin{aligned}
& \left|\left(\omega_{\xi_{n}}-\omega_{\xi_{n-1}}\right) \cdot \pi_{0}(A)\right| \leqq\left\|\left[A, a\left(\Phi_{n}\right)^{*}\right]\right\|=\left\|\left[A, \Psi\left(F_{n}\right)^{*}\right]\right\| \\
& \quad=\left\|\left[A, \Psi\left(\chi_{V} F_{n}\right)^{*}\right]\right\| \leqq 2\|A\| \cdot\left\|\Psi\left(\chi_{V} F_{n}\right)\right\|, \quad A \in \mathfrak{B}(\mathcal{O}) ;
\end{aligned}
$$

where $\chi_{V}$ is the characteristic function of the space volume $V$ which is the basis of $\mathcal{O}$ at $x_{0}=0$, and $\chi_{V} F_{n}(x)=\delta\left(x_{0}\right) f_{n}(\vec{x}) \chi_{V}(\vec{x})$; we used the commutativity of fields with observables at spacelike distances. By (2.6), (A.1) and (A.3) we have

$$
\left\|\Psi\left(\chi_{V} F_{n}\right)\right\|^{2} \leqq\left\|\chi_{V} \check{\Phi}_{n}\right\|^{2}+\left\|\chi_{V} \hat{\Phi}_{n}\right\|^{2}
$$

where $\check{\Phi}$ denotes the inverse Fourier transform, and the norm of $L^{2}\left(\mathbb{R}^{3}, d^{3} x\right) \otimes \mathbb{C}^{4}$ is used on the right.

If $f, g \in L^{2}\left(\mathbb{R}^{3}, d^{3} s\right)$ and $\hat{f}=\chi_{\Delta} \hat{f}$ with $\chi_{\Delta} \in L^{2}\left(\mathbb{R}^{3}, d^{3} s\right)$, we have the inequality

$$
\|f g\| \leqq(2 \pi)^{-3 / 2}\left\|\chi_{\Delta}\right\| \cdot\|f\| \cdot\|g\| .
$$

With $f=\breve{\Phi}_{n}$ or $\hat{\bar{\Phi}}_{n}$, we may choose $\Delta=\left\{\vec{k} /|\vec{k}| \leqq \varepsilon_{n}\right\}$ with $\Sigma \varepsilon_{n}<\infty$, and we get by (A.5), (A.6)

$$
\left\|\Psi\left(\chi_{V} F_{n}\right)\right\|^{2} \leqq \frac{2}{3(2 \pi)^{2}} V \varepsilon_{n}^{3},
$$

So that $\sum_{n}\left\|\Psi\left(\chi_{V} F_{n}\right)\right\|<\infty$ and by (A.4) $\omega_{\xi_{n}}$ is norm convergent on $\pi_{0}(\mathfrak{B}(\mathcal{O}))$.

Note added in proof. The results given here and in [2] can be established also for the quasilocal algebra of observables associated to the free scalar massless field, hence for any spin [7]. In a general theory where massless particles do appear and generate a complete set of asymptotic states below some mass threshold [3], one can construct positive energy representations of the algebra of all quasilocal observables which are normal relative to the vacuum on each future (or past) light cone, and generate a type $\mathrm{II}_{\infty}$ respectively type III $_{\lambda}$ injective factor, for each $0<\lambda<1$ [8]. Such a representation is obtained by prescribing its asymptotic configuration of outgoing massless particles to be given by a state over the outgoing field algebra constructed by a method similar to the one discussed here (or in [7] in the case of Bosons). Therefore, these results apply also to quantum electrodynamics.

\section{References}

1. Doplicher, S. : Fock representation and massless particles. Commun. Math. Phys. 3, 328 (1966)

2. Doplicher, S., Spera, M. : Representations obeying the spectrum condition. Commun. Math. Phys. 84, 505 (1982) 
3. Buchholz, D.: The physical state space of Quantum Electrodynamics. Commun. Math. Phys. 85, 49 (1982)

4. Fröhlich, J.: unpublished; Fröhlich, J., Morchio, G., Strocchi, F.: Charged sectors and scattering states in quantum electrodynamics. Ann. Phys. 119, 241 (1979)

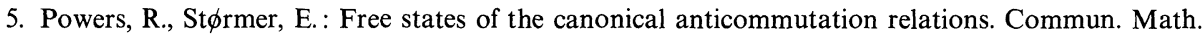
Phys. 16, 1 (1970)

6. Borchers, H. J.: On the converse of the Reeh-Schlieder theorem. Commun. Math. Phys. 10, 269 (1968)

7. Doplicher, S., Figliolini, F., Guido, D.: Infrared representations of free bose fields. Preprint

8. Buchholz, D., Doplicher, S.: Exotic infrared representations of interacting systems. Preprint.

Communicated by K. Osterwalder

Received October 19, 1982 
